\title{
Third Molar Eruption Mechanisms and Patterns
}

Winnie Zhang ${ }^{1}$

${ }^{1}$ University of Pittsburgh School of Dental Medicine, Pittsburgh PA, USA

\section{Abstract}

Third molars are highly variable in their presence and form. This report focuses on a horizontally impacted third molar and analyzes the potential etiology of this situation. Upon a clinical and radiographic examination, it was noted that the patient had four third molars present. The patient's third molars began erupting around the age of 19. Currently, they are asymptomatic with incipient caries on the occlusal surfaces. While three of the third molars erupted in a normal orientation, one of the third molars (mandibular left) erupted in an orientation that would be classified as horizontal and/or mesio-angular. No treatment has been undertaken at the moment, however surgical extraction can be recommended with the prognosis being very good. For the clinician that has to treat dental complications that arise from abnormal tooth eruption, as seen in numerous genetic and acquired disorders, knowledge about the basic molecular mechanisms involved may become extremely important.
Citation: Zhang, W. (2016) Third Molar Eruption Mechanisms and Patterns. Dentistry 3000. 1:a001 doi:10.5195/d3000.2016.49

Received: May 21, 2016

Accepted: June 13, 2016

Published: September 28, 2016

Copyright: (02016 Zhang, W. This is an open access article licensed under a Creative Commons Attribution Work 4.0 United States License. Email:wiz5@pitt.edu

\section{Introduction}

Third molars, often referred to as wisdom teeth, are the most distal (posterior) teeth of the three molars in each quadrant of human dentition. They generally erupt between the ages of 17and 23. Ideally, the third molars should erupt just like all the other teeth and be properly aligned. However, often these third molars are misaligned which can on occasion lead to crowding of the teeth or damage to adjacent teeth, and rarely to cystic pathology or root resorption [1].

Third molars can also be impacted which means they are enclosed within the soft tissue and/or jawbone or they only par- tially break through/erupt through the gum. Partial eruption of third molars provides an opening for bacteria to enter around the tooth. This could potentially cause an infection, which results in pain, swelling, jaw stiffness, and other complications. Partial eruption also makes the third molar more susceptible to caries and periodontal disease because they are in hard to reach areas, thus making it hard to brush and floss those areas [1].

There are multiple ways to classify impacted third molars. One way is based on the nature of the overlying tissues. Based on the nature of the overlying tissue impaction, impacted lower wisdom teeth can be classified into [2]:

1. Soft Tissue Impaction. When the height of the tooth's contour is above the level of the surrounding alveolar bone and the superficial portion of the tooth is covered only by soft (though this can be dense and fibrous) tissue. Soft tissue impaction is usually the easiest type of impacted tooth to remove.

2. Hard Tissue ('Bony') Impaction. This is where the wisdom tooth fails to erupt due to being obstructed by the overlying bone. This can be sub-divided into (a) partial and (b) complete bony impactions. 
a. Partial Bony. The superficial portion of the tooth is covered only by soft tissue but the height of the tooth's contour is below the level of the surrounding alveolar bone. Apart from cutting the gingiva (gum) and possible bone removal from behind the tooth, the tooth's roots may need to be divided.

b. Complete Bony. The tooth is completely encased in bone so that when the gingiva is cut and reflected back, the tooth is not seen. Bone removal (large amounts) together with root sectioning will be needed to remove the tooth. These are often the most difficult teeth to remove.

Another way to classify impacted third molars is using Winter's Classification which is based on the inclination of the impacted third molar to the long axis of the second molar [2]. Each type of impaction has some definite path of withdrawal of the teeth. Mesially impacted teeth are (can be) easier to remove whereas distally impacted teeth are (can be) the hardest to remove. Bucally positioned maxillary teeth are eas-

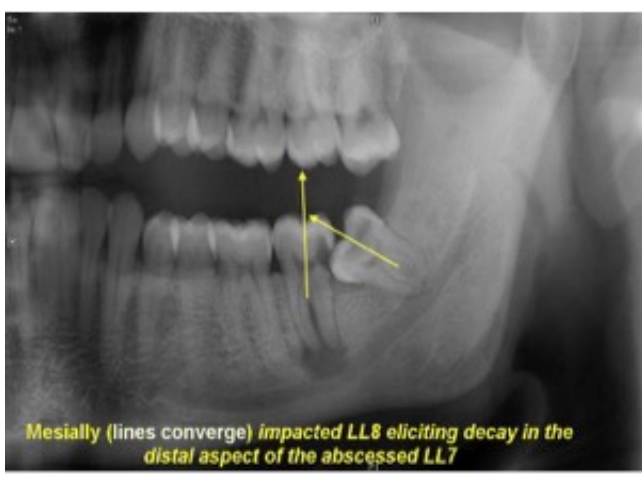

Figure 1. Mesio-angular impacted third molar (modified from [2]). ier to remove as the bone covering the tooth is thinner whereas the palatally positioned tooth requires bone removal and hence makes the extraction difficult [2].

\section{Mesio-Angular (Figure 1):}

The impacted tooth is tilted toward the 2 nd molar in a mesial direction.

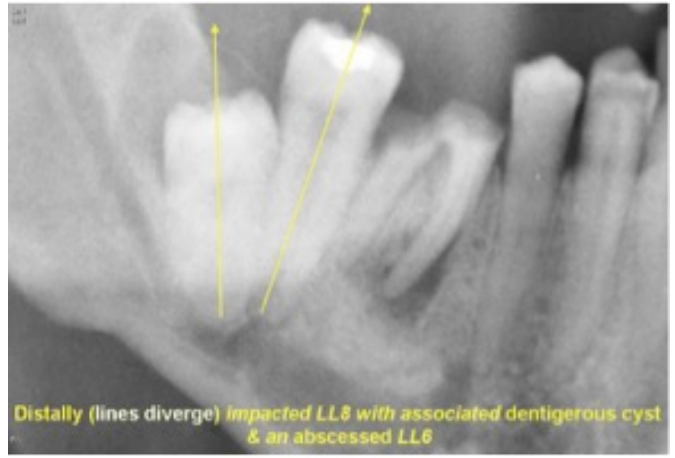

Figure 2. Disto-angular impacted third molar (modified from [2]).

2. Disto-Angular (Figure 2): The long axis of the 3rd molar is angled distally or posteriorly away from the second molar.

\section{Horizontal (Figure 3): The} long axis of the third molar is horizontal.

4. Vertical (Figure 4): The long axis of the third molar is parallel to the long axis of the second molar.

\section{Buccal / Lingual} Obliquity (Figure 5): The tooth can be buccally (tilted towards the cheek) or lingually (tilted towards the tongue) impacted.

6. Transverse (Figure 6): This type is where the tooth is in effect horizontally impacted but in the cheek or tongue direction.

7. Inverse (Figure 7): Orientation of the impacted tooth in completely inverted in reference to the occlusaly immediate opposite tooth.

\section{Familial Aggregation}

Observations of a patient and family members:

A 23 year-old Asian female with no significant medical history has received regular dental check-ups every six months throughout her life and had orthodontic treatment from age 14 to16. The patient has class I occlusion. Upon a clinical and radiographic examination, it was discovered that the patient had four third molars present (see Figure 8). The patient's third molars began erupting around the age of 19. Currently, they are asymptomatic with incipient discoloration on the occlusal surfaces. While three of the third molars erupted in a normal orientation, one of the third molars (mandibular left) erupted in an orientation that would be classi-

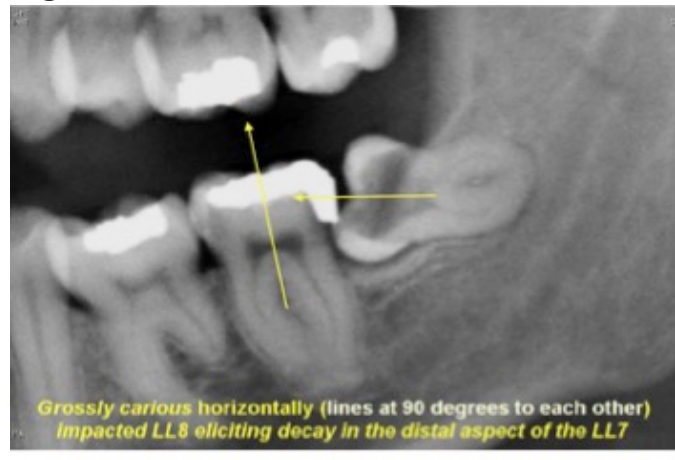

Figure 3. Horizontally impacted third molar (modified from [2]).

fied as horizontal and/or mesioangular. 


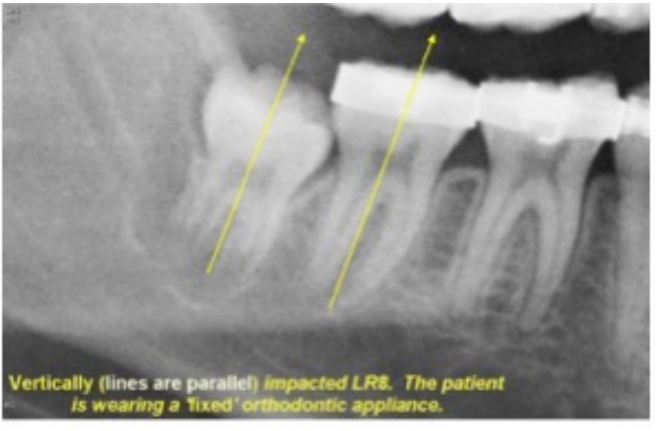

Figure 4. Vertically impacted third molar (modified from [2]).

The patient's father had four erupted third molars, all with normal eruption orientation. The patient's mother has congenitally missing third molars. The patient has a younger sibling who is 19 and third molars have not erupted but one of them is slightly mesially tipped, and impacted (Figure 9).

If one considers third molar impaction and agenesis as variation of the same clinical presentation, this family would fit an autosomal dominant mode of inheritance, suggesting a major gene effect.

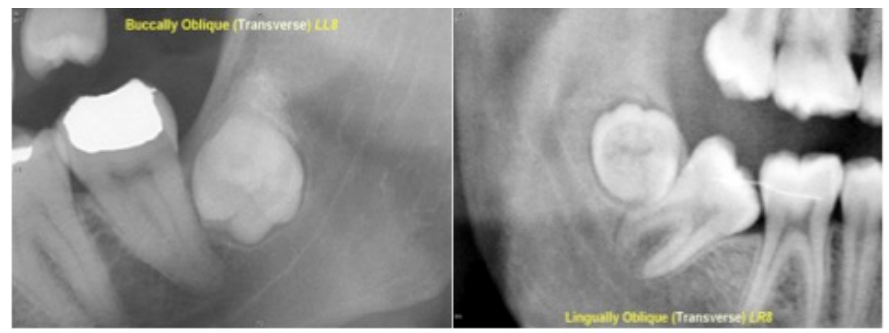

Figure 5. Lingually (left) or buccally (right) impacted third molars (modified from [2]).

\section{Mechanisms Controlling Tooth Eruption}

The etiology of the mechanisms behind eruption patterns of third molars is currently not very well understood. Tooth eruption is a complex and tightly regulated process that involves cells of the tooth organ and the surrounding alveolus. The genes that are involved in the process of tooth eruption are found all over the human genome.

Mononuclear cells (osteoclast precursors) must be recruited into the dental follicle prior to the onset of eruption. These cells, in turn, fuse to form osteoclasts that resorb alveolar bone, forming an eruption pathway for the tooth to exit its bony crypt [3]. There are many different genes that are involved in tooth eruption. Some of the molecules possibly involved in the signaling cascades of eruption have been proposed in studies from null mice, osteopetrotic rodents, injections of putative eruption molecules, and cultured dental follicle cells. In particular, recruitment of the mononuclear cells to the follicle may require colony-stimulating factor-one (CSF-1) and/or monocyte chemotactic protein-1 (MCP-1) [3]. If the recruitment of mononuclear cells to the follicle were misguided and the dental follicle of a third molar were disoriented to begin with, it could potentially result in the tooth erupting in a horizontal orientation.

Paracrine signaling by parathyroid-hormone-related protein and interleukin- $1 \alpha$, produced in the stellate reticulum adjacent to the follicle, has also been found to potentially play a role in regulating eruption [3]. This is an example of how a gene seemingly unrelated to tooth development can influence a very crucial step in dentition development. Therefore, it is

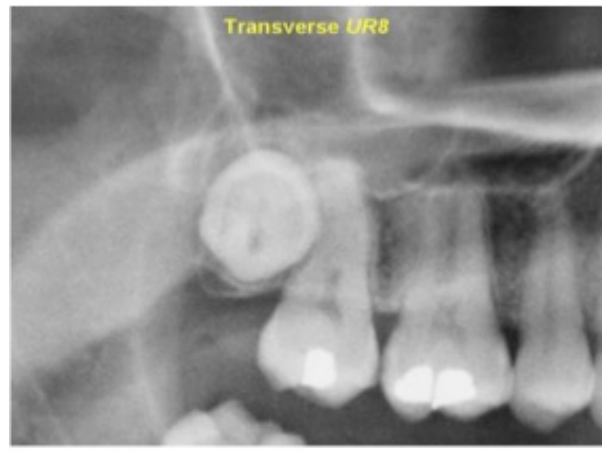

Figure 6. Transversally impacted third molar (modified from [2]).

possible that a mutation anywhere along the genome can easily influence a tooth to develop and erupt in an abnormal pattern such as horizontal eruption.

Osteoblasts might also influence the process of eruption, the most important physiologic role likely being at the eruptive site, in the formation of osteoclasts through signaling via the RANKL/OPG pathway. If this signaling pathway were interrupted by another protein, osteoblasts function could potentially be altered and interfered with. This could subsequently affect how bone was deposited. If bone was deposited only underneath the distal portion of the third molar, it could potentially cause the tooth to tip over, giving it the horizontal orientation it is in now. Evidence thus far supports a role for an osteoblast-specific transcription factor, CBFA1 (RUNX2), in molecular events that regulate tooth eruption [3]. In the case presented 


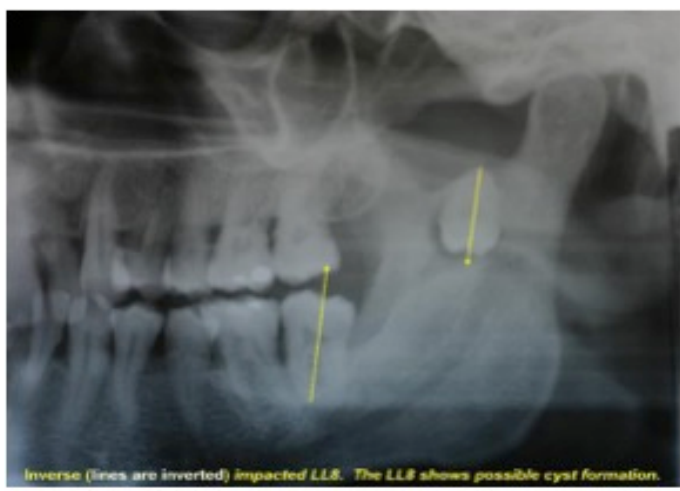

Figure 7: Inverted impacted third molar (modified from [2]).

above, one possibility is that since neither of the patient's parents had any third molar eruption abnormalities, the genetic factors that caused this horizontal orientation was probably a polygenic manifestation that resulted from a unique combination of the parents' genetic make-up. The patient's mother had congenitally

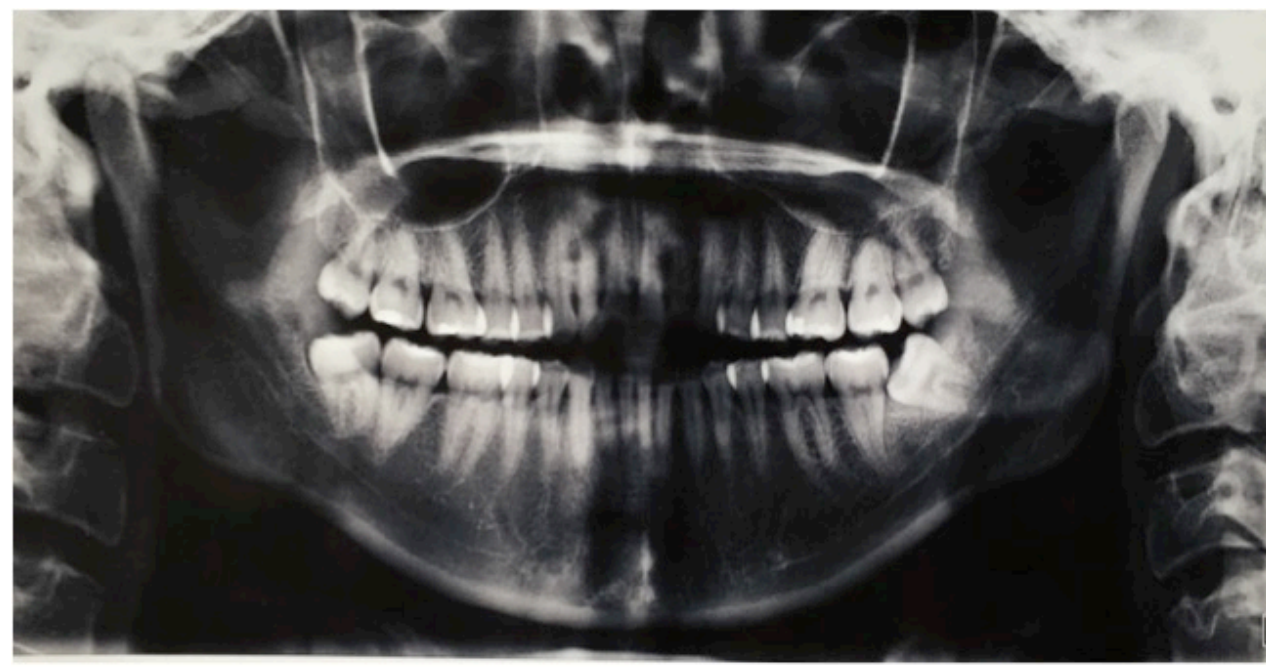

Figure 8. Panoramic radiograph shows the four third molars presents, with the left mandibular one impacted.

missing third molars, so it is possible that some of the genes which contribute to missing third molars also play a role in tooth eruption and influenced the eruption of the horizontal third molars in her offspring. The etiology of third molar impaction in the population is most likely complex with multiple

genes influencing the final outcome of a horizontally oriented third molar, whereas the case presented here exemplifies one of the instances a single gene form of inheritance is in play.

Hence, an alternative hypothesis for the etiology of this case could be that the horizontal impaction of the third molar is a variation of tooth agenesis. The mother of the patient described in this case exhibits agenesis of the third molars. The agenesis alleles could have been passed on to the patient, which then exhibited a variable phenotype of agenesis: horizontal impaction of the third molar. This hypothesis is further supported by the fact that the patient's sister 


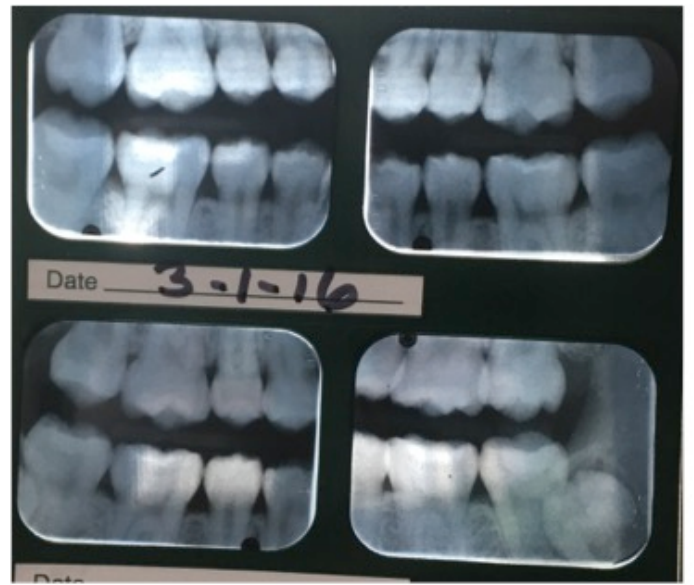

Figure 9. Bitewing radiographs of case patient's sibling.

tion of the horizontal third molar. Third molars often cause crowding in the mouth and therefore are recommended for extraction. Individuals with impaction showed more moderate to extreme crowding than those with agenesis [7]. In addition, the horizontal eruption of the mandibular left third molar has caused the mesial marginal ridge of the tooth to come in contact with the distal cervical portion of the crown on the mandibular left second molar. The orientation of the second and third left mandibular molars creates a food trap that could potentially lead to caries on the distal of the second molar or periodontal

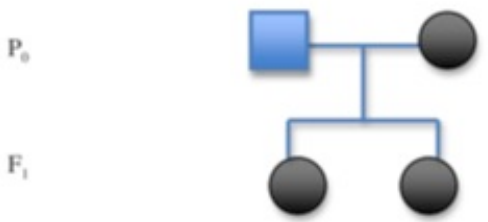

Figure 10. Pedigree of the present case. Squares are males, circles females. Blue indicates unaffected individuals, black affected.

inflammation. Therefore, a possible treatment for this case is surgical extraction of the third molar.

\section{Discussion and Practical Implications}

While the mechanisms and etiology of third molar eruption are not well understood currently, conducting more research in this aspect can yield benefits for both the field of dentistry as well as medicine as a whole. Skeletal biologists view the process of tooth eruption as a valuable model to study bone remodeling, since the emergence of a tooth into the oral cavity involves both coupled and uncoupled bone turnover events [3]. For cell and molecular biologists, tooth eruption provokes several questions concerning the tightly programmed series of signaling interactions between cells of the connective tissue sac surrounding the tooth (dental follicle) and the surrounding alveolus [3].

For a clinician that has to treat dental complications that arise from abnormal tooth eruption, as seen in numerous genetic and acquired disorders, knowledge about the basic molecular mechanisms involved is extremely important. Knowing the etiology can not only help the clinician formulate a better treatment plan for the patient, but it can help dentists better educate the patient in their dental health.

\section{References}

1. Prevalence of impacted teeth and associated pathologies--a radiographic study of the Hong Kong Chinese population. Chu FC, Li TK, Lui VK, Newsome PR, Chow RL, Cheung LK. Hong Kong Med J. 2003 Jun;9(3):158-63. PMID:12777649.

2. Mandibular third molar impaction: review of literature and a proposal of a classification.

Juodzbalys G, Daugela P. J Oral Maxillofac Res. 2013 Jul 1;4(2):e1. PMID:24422029.

3. Cellular, molecular, and genetic determinants of tooth eruption. Wise GE, Frazier-Bowers S, D'Souza RN. Crit Rev Oral Biol Med. 2002;13(4):323-34. PMID:12191959.

4. Patterns of third-molar agenesis and associated dental anomalies in an orthodontic population. Celikoglu M, Bayram M, Nur M. Am J Orthod Dentofacial Orthop. 2011 Dec;140(6):856-60.

PMID:22133951.

5. Third molar agenesis and size reduction of the remaining teeth. Garn SM, Lewis AB, Kerewsky RS. Nature. 1963 Nov 2;200:488-9. PMID:14076752.

6. Third molar agenesis and variation in size of the remaining teeth. Garn SM, Lewis AB, Kerewsky RS. Nature. 1964 Feb 22;201:839. PMID:14161232.

7. Third molar impaction and agenesis: Influence on anterior crowding. Esan T, Schepartz LA. 
Vol 4, No 1 (2016) DOI 10.5195/d3000.2016.49

Ann Hum Biol. 2016 Feb 8:1-29.

PMID:26856343. 